\title{
An Investigation on Safety Accidents in Outdoor Sports on the Perspective of Ethics in China
}

\author{
Jinfeng Chen \\ Sports Department, Changzhou Liu Guojun Higher Vocational and Technical School, Changzhou, China \\ Email: chenjf@163.com
}

How to cite this paper: Chen, J.F. (2018) An Investigation on Safety Accidents in Outdoor Sports on the Perspective of Ethics in China. Open Journal of Social Sciences, 6, 113-118.

https://doi.org/10.4236/jss.2018.64010

Received: March 19, 2018

Accepted: April 20, 2018

Published: April 23, 2018

Copyright (C) 2018 by author and Scientific Research Publishing Inc. This work is licensed under the Creative Commons Attribution International License (CC BY 4.0).

http://creativecommons.org/licenses/by/4.0/

(c) (i) Open Access

\begin{abstract}
With the rapid rise of outdoor sports in our country, the number of casualties increased dramatically. This article studies the moral issues behind the outdoor sports casualties in China from the perspective of ethics by using the literature data method and the investigation method. Starting from three perspectives: Individual participant, group participant and event organizer; this study looks for ethical issues in an accident, and discusses the causes. Furthermore, the author further analyzes the moral norms that our country outdoor sports should follow, and suggests that the following practices should be done to promote the harmonious developments of outdoor sports in China: 1) Strengthen moral education and the unity of knowledge and practice. 2) Improve self-discipline and improve personality. 3) Strengthen guidance of social public opinions, and value guidance. 4) Strengthen the moral evaluation and cultivation.
\end{abstract}

\section{Keywords}

Ethics, Perspective, Outdoor Sports, Accident, Investigation

\section{Introduction}

With the rapid development of China's economy and technology, outdoor sports are being recognized and accepted by more and more people as a new movement. At present, China's outdoor sports have the dual characteristics of extreme sports and public leisure. All kinds of mountaineering, hiking, cycling club, QQ group, WeChat group have mushroomed. According to relevant statistics, the number of outdoor sports participation in China has reached 130 million, taking up about $10 \%$ of the country's population. The industry has reached RMB 147.7 billion [1]. But with it a sharp increase comes in the number of outdoor accidents in China. According to the Chinese mountaineering association, 
160 accidents occurred in the national mountaineering outdoor sports in 2014, and the number of dead and missing has nearly doubled compared with 2013. It is not difficult to find out from the cases of death and disappearance that almost all cases are caused by human factors which can be avoided or prevented [2]. From the perspective of ethics, this paper discusses the humanistic and moral factors behind outdoor sports casualties in China, analyzes the moral norms that should be followed in the healthy development of outdoor sports in China and puts forward the moral countermeasures to promote the harmonious development of outdoor sports in China in order to provide some reference for the participants, organizers and managers of outdoor sports in China. With the moral problems in the process of outdoor sports in China as the object of study, this paper studies the current outdoor participants, the ethical status of the organizer, the causes and the relevant experts in the field of interview. It uses two research methods. One is literature review method. This paper consults relevant literature in CNKI, and it collects and organizes the literature in the libraries of Changzhou University and NHTV Breda University of Applied Sciences. The other one is questionnaire method. There are participants questionnaire and organizer questionnaire. This paper mainly investigates participants' participation motivations, moral standards, action rules, environmental status and moral evaluations, and organizers' principles, group composition, motivations, codes of action and moral evaluations [3].

\section{Research Results and Analysis}

\subsection{The Moral Status of Outdoor Sports in China Is Shown in Figure 1}

Through investigation on the status of outdoor sports ethics, this paper finds that the six categories of problems with a selection rate of over $50 \%$ can be summarized into three aspects: Individual participants, group participants, and outdoor sports organizers.

Individual participants are mainly represented in "freedom of actions" and "neglecting life and environmental responsibility". The purpose of participation in outdoor sports is the pursuit of freedom to a large extent. But outdoor sports are often associated with greater risks. So, the freedom of outdoor movements should be relative freedom and must be based on the rules. Look at the outdoor accidents, there always are problems of following their inclinations and unfettered participants [4].

Group participants were mainly shown in the two aspects of "lack of trust" and "lack of personal care". Trust is the key to interpersonal communications and the foundation of a harmonious society. Most outdoor sports are organized as teams, which requires team members to communicate and help each other. The mutual trust between members is the premise to maintain the team and complete the task. If the team members are not fully trusted, the team's synergy and cohesion will decline and the team interests and processes of outdoor activities will be jeopardized. 


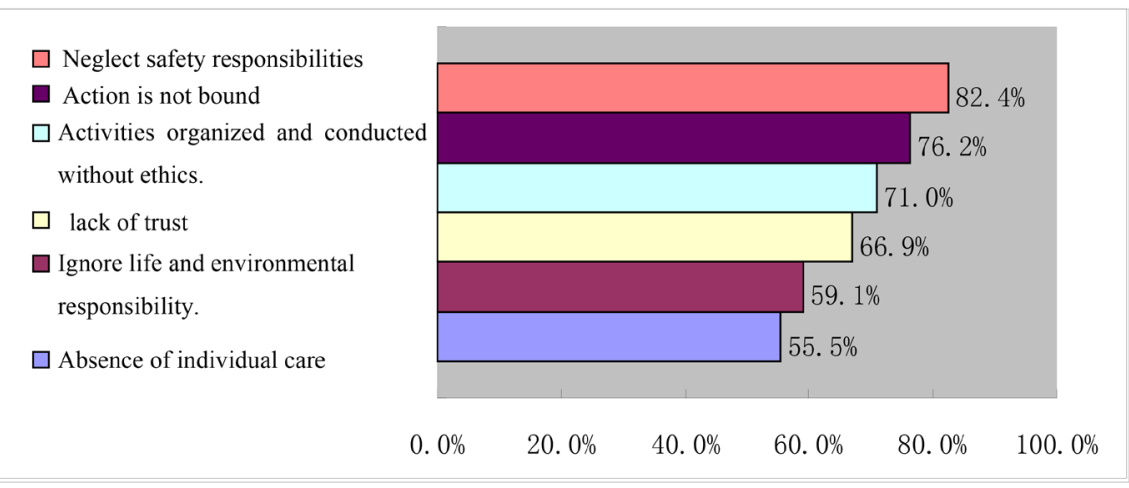

Figure 1. The moral status of outdoor sports in China.

The organizers of outdoor sports are mainly concerned with "neglecting safety responsibility" and "organizing and carrying out immoral principles". At present, due to the short time of outdoor sports entering China, there has not been a standardized system and ethics. The huge Chinese outdoor market is full of motley clubs, groups and private clubs. It's a very scary thing to organize various kinds of outdoor groups by organizing acquaintances or online friends in the absence of institutions and regulations, lines planning and reasonable arguments without considering the outfitting of outdoor professional personnel and professional equipment.

\subsection{The Causes of Moral Problems of Outdoor Sports in China Is Shown in Figure 2}

According to the survey, the causes of outdoor sports ethics can also be summarized into three aspects: Participants themselves, organizers and social reasons.

The participants themselves were mainly concerned with "lack of attention to activity experience", "curiosity" and "little contact with the natural environment". They perform with absence of moral consciousness. And they are above predecessors' experience, not listen to the explanation carefully, not study the technology carefully. They think there must have no problem, and their hearts are arrogant. But because of the curiosity and regardless the dissuasion, they always want to try to risk. All above result in discord in the team and prone accidents. Modern people are used to living in reinforced concrete cities, and have too little contact with nature, so they Lack necessary field survival skills. The lack of knowledge and awe in nature is a manifestation of the expansion of personal desires [5].

The main reasons for organizers are "lack of professional responsibility", "lack of moral education" and "poor safety awareness". The specific performance is lack of moral accomplishment. At present, the organization and development of outdoor activities in China mainly rely on private clubs and industry associations, and a small number of network groups. The organizers are not clear about their rights and obligations. They often organize and manage the outdoor sports according to hobbies and business needs. They only aim to carry out activities 
Lack of professional responsibility. $\square$ Lack of attention to activity experience. Big Curiosity

$\square$ Not sound moral system

$\square$ Lack of a moral evaluation system.

$\square$ Moral education lags behind.

$\square$ Not strong safety awareness.

$\square$ Not strong public opinion

$\square$ Little contact with the natural environment.

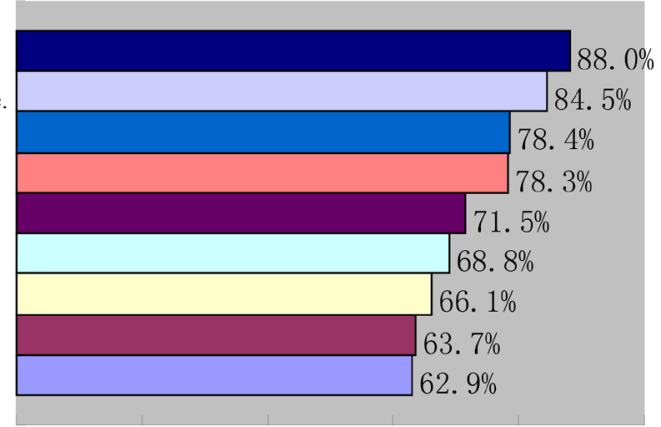

$\begin{array}{llllll}0.0 \% & 20.0 \% & 40.0 \% & 60.0 \% & 80.0 \% & 100.0 \%\end{array}$

Figure 2. The causes of moral problems of outdoor sports in China.

and have no moral quality at all. And after the team was organized, there is no awareness of ethics and safety education.

The social reasons include "unsound moral system", "lack of moral evaluation system" and "weak public opinion". At present, there are thousands of outdoor clubs in China, but quite a few of them are not regulated. They have neither relevant professional outdoor sports technology, safety instructors, nor related professional sports equipment. There are no specific professional ethics rules in the industry. Relevant functional departments are also difficult to manage effectively. In the activity, the uncertainty of the responsibility subject is the most typical characteristic of the lack of moral evaluation system. The not strong social public opinion leads to the results that the people do not understand the operation mode of the club and are not clear what kind of people are organizing and managing. Between the organizer and the participants, the participants were always in a weak position. When interests are damaged, most people choose to admit their own misfortune rather than pursue specific reasons. Because the whole process of investigation will consume more benefits than loss of interest, such as money, time and experience [6].

\subsection{The Codes of Ethics for Outdoor Sports in China Is Shown in Figure 3}

According to the survey, organizing in order (89.0\%), trusting and obeying the team (85.0\%), understanding teammates (82.5\%), keeping self discipline (80.0\%), helping teammates (77.1\%) and taking away the trash $(65.8 \%)$ are chosen as the six moral codes to be followed for outdoor sports in China. These reflect the individual's good wishes for others, the groups and the nature, and also reflects the individual's willingness to accept responsibilities and obligations.

The "organizing in order" ranking first shows the psychological expectations of most participants to the organizer's sense of responsibility and organizational management ability. The organizers should take full account of all contingencies and details from the beginning of the itinerary planning, and should master each person's individual characteristics and abilities. They also should know equipment and skills required for the activity [7]. 


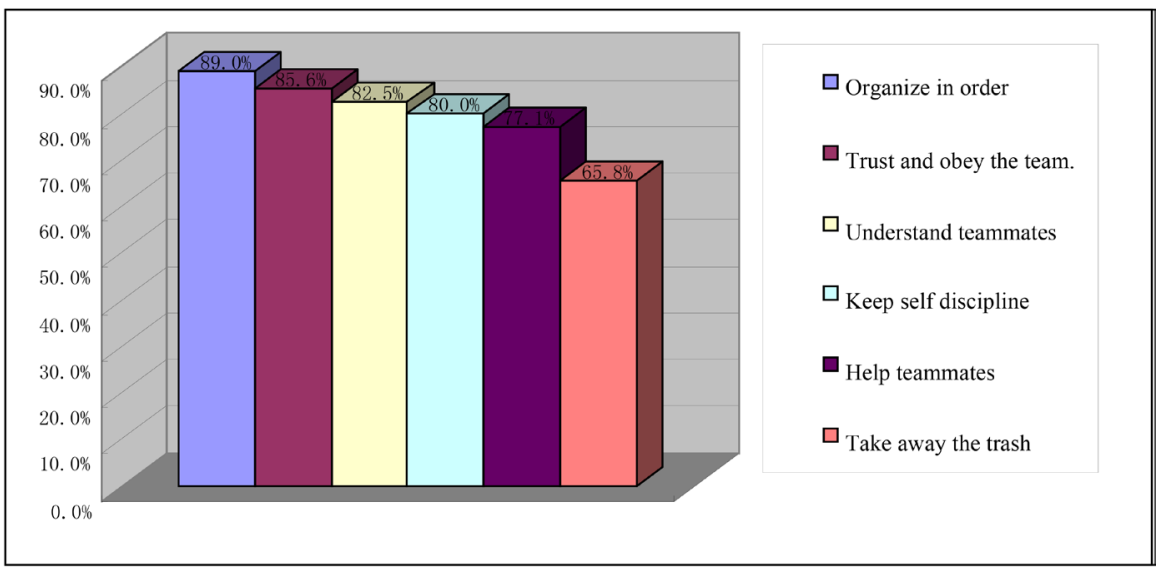

Figure 3. The codes of ethics for outdoor sports in China.

\section{The Moral Countermeasures to Promote the Harmonious Development of Outdoor Sports in China}

First, strengthen moral education and the unity of knowledge and practice. Outdoor activities are organized and planned activities. The organizers should carry out the following three aspects of ethics education before the event. 1) Carry out life ethics education to enhance the safety awareness of participants. 2) Carry out ethics education to promote the harmonious and orderly relationship among the team members. 3) Carry out environmental ethics education to promote the ecological consciousness of the participants in order to protect the environment.

Second, improve self-discipline and personality. Moral cultivation refers to people's self-reform, self-training and self-cultivation in the aspects of moral qualities, moral emotions, moral wills and moral habits. Individual improvement of moral cultivation should include two aspects: One is to demand oneself with good ethics. In daily life, we should constantly improve our personal moral standards and not do anything unethical. The second is to regulate and maintain the fine quality, sentiment and moral realm formed after the activities of daily life, outdoor sports, inner and outer activities.

Third, strengthen social public opinion guidance and value guidance. Public opinions carry forward the main theme of values of respect, care, mutual assistance, unity, struggle and security, which is an important way to improve the social moral environment. The demonstration and typical effect of strengthening public figures can strengthen the sense of identity of ordinary people and carry forward the spirit of The Times of outdoor sports.

At last, strengthen the moral evaluation and cultivation. Aiming at the ethics of outdoor sports, government related functional departments should actively evaluate the moral behaviors, moral philosophies and moral standards of outdoor sports participants. To evaluate the moral behavior of outdoor participants in practical activities. They should help affect the moral behaviors of participants and organizers through evaluation of content guidance to help the broad masses 
of people to build a good outdoor sports moral environment. In addition, government departments should strengthen the accountabilities of outdoor organizers [8].

\section{Conclusion}

At present, the development of outdoor sports in China is still in the initial stage, but its pace of development is striking. Frequent accidents and disasters are related to the rapid influx of new people. According to the standards of western developed countries, there is still a lot of room for development of outdoor sports in China. In the next decade, China's outdoor population is expected to double again. There is an urgent need for a moral culture in the field of outdoor sports in China. We should adhere to the basic guiding principles of people-oriented, balanced, risk prevention and harmonious development. With the participants and organizers of outdoor activities actively performing the basic functions of power and obligation, the outdoor sports in China can solve the problem of ethical and moral issues and build a harmonious outdoor environment.

\section{References}

[1] Zuo Yu Capital (2015) Analysis of China's Outdoor Sports Tourism Industry Based on the Whole Industry Chain Perspective. http://www.lieyunwang.com/archives/137057

[2] China Mountaineering Association Accident Investigation Team (2014) Report on the Accident of Mountaineering Outdoors in Mainland China. Chinese Mountaineering Association, Beijing. 2015-5.

[3] Luo, G.J. (1988) Ethics. People’s publishing House, Beijing, 364.

[4] Tencent Website (2014) Wang Shi Recalled Climbing Mountain Everest: The Real Challenge Is Yourself. http://finance.qq.com/a/20140409/014694.htm

[5] China Youth News (2015) Similar Tragedies Are Repeated in Outdoor Sports-The Service System Was Lagging Behind. http://www.chinanews.com/ty/2015/10-30/7597206.shtml

[6] Berg, E.C., Migliaccio, T.A. and Anzini-Varesio, R. (2014) Female Football Players, the Sport Ethic and the Masculinity-Sport Nexus. Sport in Society, 17, 176-189. https://doi.org/10.1080/17430437.2013.828699

[7] Curry, T. and Strauss, R.H. (1994) A Little Pain Never Hurt Anyone: A Photo-Essay on the Normalization of Sports Injury. Sociology of Sport Journal, 11, 195-208. https://doi.org/10.1123/ssj.11.2.195

[8] Wang, Y.Z. (2011) Study on the Bayu Sports Cultures. Sports Culture Guide, 3, 36-37. 